\title{
Modelagem do Problema de Cobertura de Conjunto para Recomendação de Objetos de Aprendizagem aplicado ao Repositório do YouTube
}

\author{
Eduardo Augusto Costa Trindade - UFVJM - eduardo.trindade@ufvjm.edu.br \\ Luciana Pereira de Assis - UFVJM - lpassis@ufvjm.edu.br \\ Alessandro Vivas Andrade - UFVJM - alessandrovivas@ufvjm.edu.br \\ Henrique Carlos Fonte Boa Carvalho - UFVJM - henriquefbc@ gmail.com \\ Cristiano Grijó Pitangui - UFSJ - pitangui.cristiano@gmail.com \\ Fabiano Azevedo Dorça - UFU - fabianodor@ufu.br
}

\begin{abstract}
Resumo: Este trabalho propõe um novo modelo para o Problema de Cobertura de Conjunto utilizado no processo de recomendação de objetos de aprendizagem. Cada objeto traz um conjunto de conceitos e, para evitar uma sobrecarga cognitiva do aprendiz, é necessário atentar-se para as repetições desses conceitos. Assim, a modelagem proposta consiste na seleção de um conjunto de objetos com menor custo e menor repetição de conceitos. Para solucionar o problema foi utilizado um algoritmo genético associado à um sistema de recomendação de objetos integrado à API do YouTube. Os resultados dos experimentos demonstram que a abordagem proposta se mostra promissora, possibilitando uma recomendação eficiente de vídeos que atenda às requisições do usuário.
\end{abstract}

Palavras-chave: Recomendação; Objetos de Aprendizagem; Ensino eletrônico, YouTube.

\section{Modeling the Set Coverage Problem for Learning Object Recommendation applied to the YouTube Repository}

Abstract: This work proposes a new model for the Set Coverage Problem used in the
process of recommending learning objects. Each recommended object brings a set of
concepts and, to avoid a cognitive overload of the learner, it is necessary to pay attention
to the repetitions of these concepts. Thus, the proposed modeling consists of selecting a
set of objects with lower cost and less repetition of concepts. To evaluate the model, a
genetic algorithm associated with a system of recommendation of objects integrated with
the YouTube API was used. The results of the experiments show that the proposed approach
is promising, enabling an efficient recommendation of videos that meet the user's requests. Keywords: Recommendation; Learning Objects; E-learning, YouTube.

\section{Introdução}

Com o intuito de tornar o processo de aprendizagem mais assertivo, novas técnicas têm surgido visando auxiliar a organização, seleção e utilização de materiais que possam contribuir com êxito tanto no ensino quanto na aprendizagem. A essa nova maneira de pensar o desenvolvimento, a organização, recomendação, busca e reutilização de materiais educacionais, surge o conceito de Objetos de Aprendizagem (OAs) (BRAGA et al., 2012).

De acordo com Wiley (2002), um Objeto de Aprendizagem é qualquer recurso digital que possa ser reutilizado para suporte ao ensino. Eles são definidos como entidades, digitais ou não, que possuam em suas características principais a reutilização ou modelagem 
durante o processo de ensino apoiado pela tecnologia, tais como, conteúdos multimídia, instrucionais, software, entre outros.

As principais características de Objetos de Aprendizagem são: acessibilidade, reusabilidade, interoperabilidade, portabilidade e confiabilidade (BRAGA et al., 2012). Para facilitar o processo de organização e recuperação de informações, os OAs são organizados em Repositórios de Objetos de Aprendizagem (ROA). Um Repositório de Objetos de Aprendizagem pode ser definido como um banco de dados que armazena OAs e informações que facilitam a sua busca (metadados) (JUNQUEIRA; LOSCIO, 2014).

Quanto à acessibilidade, os OAs devem possuir metadados padronizados para facilitar seu processo de recuperação. Em relação à reusabilidade, o processo de criação deve garantir que possam ser reutilizados em diferentes contextos de aprendizagem. A acessibilidade e a usabilidade são fatores essenciais na elaboração de OAs que respeitem as especificidades humanas (MOREIRA; CONFORTO, 2011). A interoperabilidade e portabilidade garantem que os OAs possam ser utilizados em diferentes plataformas. Por fim, devem ser armazenados em meios que garantam a confiabilidade.

O Problema de Recomendação de Objetos de Aprendizagem (PROA) trata de um problema de recomendação de conteúdo baseado nos conceitos. Isto é, conteúdos curriculares são um sequenciamento de conceitos necessários para atingir os objetivos de aprendizagem, e o PROA consiste em escolher de forma otimizada os OAs que cubram estes conceitos.

Atualmente, um dos grandes e mais utilizados repositórios de objetos de aprendizagem no formato de vídeos é o YouTube. No Brasil, 9 em cada 10 usuários que acessam a plataforma do YouTube estão em busca de aprender algo novo (YOUTUBE, 2019b). Além disso, trata-se de um espaço que gera bilhões de horas de vídeos e de visualizações, sendo que mais da metade dessas visualizações são realizadas em dispositivos móveis (YOUTUBE, 2019b).

Assim sendo, neste trabalho é desenvolvido um Sistema de Recomendação de Objetos de Aprendizagem (SROA) personalizado com o conteúdo dos vídeos do YouTube. O sistema proposto não só efetua buscas no repositório, como também seleciona um conjunto mínimo que cubra todos os conceitos requisitados pelo usuário.

O processo de seleção dos vídeos é baseado em um novo modelo min-max para Problema de Cobertura de Conjunto que objetiva minimizar o custo dos objetos e o número máximo de repetições de conceitos. Essa nova abordagem contribui para evitar uma sobrecarga cognitiva do aprendiz e desestimular o aprendizado. O cálculo do custo é obtido a partir dos atributos do vídeo, como likes, dislikes e views.

Com as contribuições apresentadas, este trabalho aponta os seguintes questionamentos: a nova modelagem proposta é capaz de gerar um conjunto de objetos otimizado que, além do custo, consiga minimizar também a repetição de conceitos? O SROA proposto se adéqua ao repositório do YouTube?

O presente trabalho se organiza como segue. A Seção 2 apresenta uma revisão dos trabalhos correlatos e a definição do problema de cobertura de conjunto. O Sistema de Recomendação de Objetos de Aprendizagem proposto é apresentado na Seção 3. A Seção 4 apresenta uma análise do algoritmo genético, utilizado para solucionar o problema. A melhor configuração do algoritmo foi aplicada em simulações de uso do SROA. Por fim, são apresentadas as conclusões e sugestões de trabalhos futuros (Seção 5). 


\section{Trabalhos relacionados}

Em uma Revisão Sistemática da Literatura, Pontes et al. (2014) descrevem diferentes tipos de filtragem de conteúdo aplicada no processo de recomendação de OA: colaborativa, baseada em conteúdo, demográfica, baseada em conhecimento, baseada em utilidade, baseada em outros contextos, híbrida. O presente trabalho apresenta uma filtragem baseada em conteúdo, uma vez que evidencia os conceitos embutidos nos objetos, bem como na qualidade dos mesmos. Todavia, vale ressaltar a colaboração implícita baseada em popularidade, tendo em vista a avaliação feita por usuários nos conteúdos avaliados, conforme é demonstrado na Subseção 3.2 deste artigo.

Também como uma filtragem baseada em conteúdo, Vieira e Nunes (2012) e Cazella et al. (2012) recomendam OAs, um de acordo com as semelhanças existentes entre eles e, o outro, de acordo com perfil do aluno e nas competências que os OAs podem ajudar a construir. Com uma abordagem similar, Aguiar et al. (2015), Mendes et al. (2017), Junior e Francisco (2018) apresentam o mesmo critério de recomendação baseado no Estilo de Aprendizagem (EA) do aluno. Já Guerra et al. (2020), demonstram um protótipo de uma aplicação mobile que busca avaliar a acessibilidade de OAs, aplicando técnicas de Inteligência Artificial avaliando a acessibilidade dos mesmos no formato de texto.

Existem na literatura inúmeros trabalhos que abordam a recomendação de vídeos, como apresentado por Davidson et al. (2010) que selecionam um conjunto personalizado de vídeos baseado nas atividades do usuário. Madden et al. (2013), Pinheiro (2019) e Serbanoiu e Rebedea (2013) qualificam vídeos com base nos metadados (autor, categoria, curtidas, visualizações, comentários, dentre outros) e apresentam um ranking desses vídeos ao usuário. Nagumo et al. (2019) realizaram uma revisão sistemática da literatura chegando à conclusão que existe uma lacuna entre os estudos de nível superior sobre a busca por aprendizagem informal. Além disso, os autores mencionam o YouTube como meio para atender a uma demanda própria na busca por aprendizagem, revisão e preparação de conteúdos audiovisuais. Por fim, Barrére et al. (2020) consideram o professor como ator principal do processo de ensino, e neste contexto, desenvolveram um sistema de recomendação, conectado à plataforma do Moodle na forma de um plugin nomeado OVR (Open Video Recommendation), com o objetivo de incorporar aspectos de pesquisa e recomendação de vídeos educacionais proventes deste repositório.

Neste trabalho, os objetos de aprendizagem são vídeos do repositório do Youtube e a busca neste repositório é feita pela API do próprio Youtube. A API, que provém do Inglês Application Programming Interface, é um conjunto de rotinas e padrões estabelecidos por um software para a utilização das suas funcionalidades por aplicativos que não pretendem envolver-se em detalhes da implementação do software, mas apenas utilizar seus serviços (FOLDOC, 2015). A API de dados do YouTube permite que desenvolvedores de sistemas incorporem em seus projetos (sites, aplicativos, etc.), funções que normalmente são executadas no site do YouTube. Este serviço é gratuito e limitado (YOUTUBE, 2019a).

Seguindo uma outra perspectiva, este trabalho busca um conjunto mínimo de vídeos que atendam aos conceitos apresentados pelo usuário durante a busca. O processo de seleção é modelado como um problema de cobertura de conjunto (JUNIOR; FRANCISCO, 2018; MAVROMMATIS, 2006). A abordagem proposta neste trabalho traz inovações ao incluir outro critério de otimização além de redução do custo: a minimização das repetições de conceitos dentre o conjunto de objetos recomendados. Além disso, o sistema de recomendação de OAs proposto faz uso das características do vídeo (curtidas, visualizações, entre outros) e nos conteúdos abordados nos objetos durante o processo de seleção. 


\subsection{Problema de Cobertura de Conjunto}

Dada uma matriz binária de $m$ linhas e $n$ colunas, o Problema de Cobertura de Conjunto (PCC) consiste em cobrir as $m$ linhas por um subconjunto de colunas. Cada coluna possui um custo, não negativo, $c_{i j}$. O objetivo é encontrar um subconjunto de colunas de custo mínimo (BEASLEY; CHU, 1996).

A modelagem matemática é apresentada nas Equações 1-3.

Seja $x_{j}= \begin{cases}1, & \text { se coluna } j \text { encontra-se na solução } \\ 0, & \text { caso contrário. }\end{cases}$

$$
\text { Minimize } \sum_{j=1}^{n} c_{j} x_{j}
$$

Sujeito à:

$$
\begin{gathered}
\sum_{j=1}^{n} a_{i j} x_{j} \geq 1, i=1, \ldots, m \\
x_{j} \in\{0,1\}, j=1, \ldots, n
\end{gathered}
$$

A função objetivo (Equação (1)) consiste em minimizar o custo total associado às colunas presentes na solução. A restrição (2) garante que todas as linhas $m$ serão cobertas por pelo menos uma coluna e a restrição (3) é uma restrição de integralidade.

Neste contexto, as linhas representam os conceitos e as colunas os objetos de aprendizagem. Considere uma matriz $A_{m x n}, a_{i j} \in A$ é igual a 1 , se conceito $i$ é coberto pelo objeto de aprendizagem $j$, ou 0 , caso contrário.

Para exemplificar, dado um conjunto de conceitos $\{\mathrm{a}, \mathrm{b}, \mathrm{c}, \mathrm{d}, \mathrm{e}, \mathrm{f}\}$ e um conjunto de objetos de aprendizagem $\left\{S_{1}, S_{2}, S_{3}, S_{4}, S_{5}\right\}$, sendo que cada objeto representa um subconjunto de conceitos: $\mathrm{S}_{1}=\{\mathrm{b}, \mathrm{e}\}, \mathrm{S}_{2}=\{\mathrm{f}\}, \mathrm{S}_{3}=\{\mathrm{a}, \mathrm{b}\}, \mathrm{S}_{4}=\{\mathrm{a}, \mathrm{c}, \mathrm{d}\}$ e $\mathrm{S}_{5}=\{\mathrm{d}, \mathrm{e}, \mathrm{f}\}$. A Tabela 1 apresenta a relação entre os conceitos e objetos na situação descrita.

\section{Tabela 1. Representação do PCC por Matriz}

\begin{tabular}{|l|l|l|l|l|l|}
\cline { 2 - 6 } \multicolumn{1}{c|}{} & $S_{1}$ & $S_{2}$ & $S_{3}$ & $S_{4}$ & $S_{5}$ \\
\hline a & 0 & 0 & 1 & 1 & 0 \\
\hline b & 1 & 0 & 1 & 0 & 0 \\
\hline c & 0 & 0 & 0 & 1 & 0 \\
\hline d & 0 & 0 & 0 & 1 & 1 \\
\hline e & 1 & 0 & 0 & 0 & 1 \\
\hline f & 0 & 1 & 0 & 0 & 1 \\
\hline
\end{tabular}

É evidente que se for considerado todos os objetos $S$ é possível cobrir todos os conceitos. No entanto, os objetos $S_{1}=\{\mathrm{b}, \mathrm{e}\}, S_{2}=\{\mathrm{f}\}$ e $S_{4}=\{\mathrm{a}, \mathrm{c}, \mathrm{d}\}$ também atendem essa restrição. Outra solução possível seria recomendar os objetos $S_{3}=\{\mathrm{a}, \mathrm{b}\}, S_{4}=\{\mathrm{a}, \mathrm{c}, \mathrm{d}\}$ e $S_{5}=\{\mathrm{d}, \mathrm{e}, \mathrm{f}\}$. Verifica-se que esta solução apresenta repetidamente o conceito $a$, nos objetos $S_{3}$ e $S_{4}$, bem como o conceito $d$ ao recomendar os objetos $S_{4}$ e $S_{5}$.

Avaliando as diferentes soluções, no contexto da recomendação de objetos de aprendizagem, percebe-se que algumas delas podem acarretar em uma sobrecarga cognitiva do aprendiz, uma vez que um mesmo conteúdo será abordado em vários objetos. Sendo 
assim, este trabalho propõe uma nova modelagem para o problema de cobertura de conjunto que busca, além da redução do custo, minimizar o número máximo de repetições de um determinado conceito. A Seção 3 apresenta a descrição do sistema proposto com a nova modelagem do PCC.

\section{Sistema de Recomendação de Objetos de Aprendizagem}

O sistema de recomendação de objetos de aprendizagem apresentado neste trabalho utiliza como repositório o Youtube. Porém, o modelo proposto é extensível para diversos outros repositórios disponíveis na web. Foram utilizadas as seguintes tecnologias na prototipação: Vue.js (framework do JavaScript de código aberto para a construção de interfaces de usuário) e CSS (Bootstrap v4.3.1).

O sistema representado na Figura 1 apresenta o ciclo do SROA em questão. Inicialmente, o usuário interage com o sistema padronizando sua busca. A consulta é realizada na API do YouTube e o resultado é filtrado de acordo com os conceitos pré-definidos. Posteriormente, o conjunto de objetos retornados passa para a fase de seleção, modelado como um Problema min-max de Cobertura de Conjuntos, proposto neste trabalho (subseção 3.1), para minimizar o custo e repetições dos conceitos para recomendação adequada dos OAs.

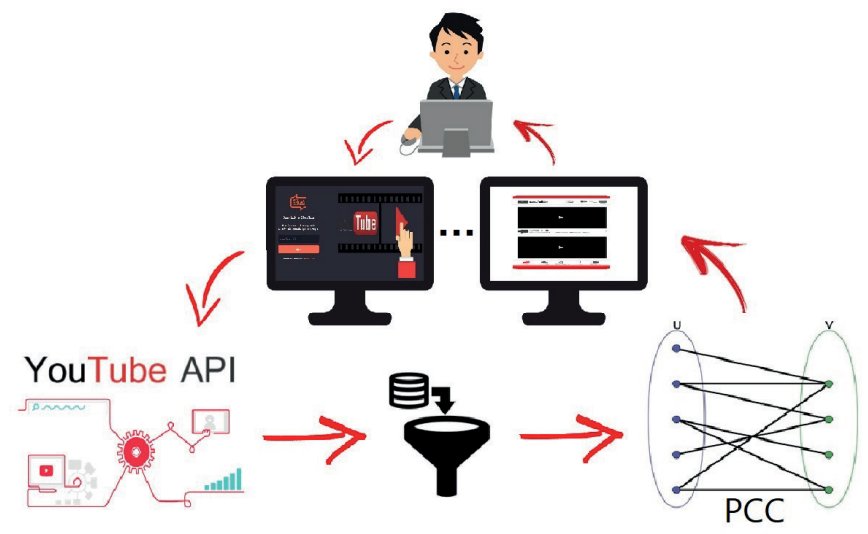

Figura 1. SROA: Processo de busca, filtragem e retorno de OAs

Os custos dos objetos de aprendizagem podem ser definidos de diferentes formas, dependendo das informações disponíveis e do contexto aplicado. Junior e Francisco (2018), por exemplo, definiram o custo com base nas características do aprendiz, baseando-o no estilo de aprendizagem do estudante. Neste trabalho, o custo é obtido a partir dos atributos do vídeo (Subseção 3.2). Após definida a função de custo, um algoritmo genético é utilizado para solucionar o problema min-max de cobertura de conjunto e encontrar o conjunto mínimo de objetos de aprendizagem que abordam todos os conteúdos requisitados pelos usuários. Por fim, este conjunto é exibido ao usuário.

\subsection{Novo Modelo Min-Max de Cobertura de Conjunto}

Após avaliar as inconveniências encontradas com o uso de uma modelagem tradicional do problema de cobertura de conjunto, essa Seção apresenta um novo modelo matemático min-max no qual, além do custo, busca minimizar o número máximo de repetições de conceitos dentre os objetos selecionados.

O modelo proposto é uma extensão do PCC. As modificações implementadas no modelo são apresentadas nas Equações 4-6. 
- Variável adicional:

- $r_{\max }=$ número máximo de repetições que uma coluna é coberta por diferentes linhas

- Função Objetivo:

$$
\text { Minimize } \sum_{j=1}^{n} c_{j} x_{j}+r_{\max }
$$

- Restrições adicionais:

$$
\begin{gathered}
\sum_{j=1}^{n} a_{i j} x_{j} \leq r_{\max }, i=1, \ldots, m \\
r_{\max } \geq 1
\end{gathered}
$$

A função objetivo (4) consiste em minimizar o custo total associado às colunas (que representam os OAs) presentes na solução, somado à variável $r_{\max }$. Essa variável, adicionada à função objetivo, garante que o número máximo de vezes que as linhas (que representam os conceitos) são cobertas por diferentes colunas (OAs) seja mínimo. A restrição (5) indica que cada linha (conceito) deve ser coberta por no máximo $r_{\max }$ colunas (OAs). A restrição (6) representa a restrição de não-negatividade..

O custo $c_{i j}$ das linhas, que neste contexto representam objetos de aprendizagem, é calculado de acordo com os atributos do vídeos retornados pela API do YouTube, conforme descrito na Subseção 3.2.

\subsection{Composição do custo dos Objetos de Aprendizagem}

A avaliação da qualidade dos objetos retornados após busca na API do Youtube é calculada a partir dos atributos apresentados na Tabela 2. Esses atributos se mantém constantemente atualizados de acordo com as preferências dos usuários, incorporando ao sistema proposto o aspecto de filtragem de conteúdo colaborativa.

Tabela 2. Atributos considerados na avaliação do custo

\begin{tabular}{l|l}
\hline Atributo & Definição \\
\hline Título & Descrição resumida do conteúdo apresentado \\
\hline Views & Número de visualizações do vídeo \\
\hline Likes & Quantitativo de avaliações positivas do vídeo \\
\hline Dislikes & Quantitativo de avaliações negativas do vídeo \\
\hline
\end{tabular}

Inicialmente, calcula-se a média de likes, dislikes e views da amostra $n$ de vídeos encontrados. Essa amostra pode ser alterada conforme a necessidade, mas utiliza-se $n=50$ que é o valor máximo retornado em uma consulta com consumo de quotas livres fornecidas pela API do YouTube (YOUTUBE, 2019a). Com as médias encontradas, calcula-se o custo de cada vídeo (objeto de aprendizagem), conforme Tabela 3.

Tabela 3. Definição dos custos das variáveis

\begin{tabular}{l|l|l}
\multicolumn{1}{c|}{ Likes } & \multicolumn{1}{c|}{ Dislikes } & \multicolumn{1}{c}{ Views } \\
\hline $\mathrm{x} \leftarrow$ mediaLikes & $\mathrm{y} \leftarrow$ mediaDislikes & $\mathrm{z} \leftarrow$ mediaViews \\
se numeroLikes $>=\mathrm{x}$ & se numeroDislikes $<=\mathrm{y}$ & se numeroViews $<=\mathrm{z}$ \\
entao custoLikes $\leftarrow 0$ & entao custoDislikes $\leftarrow 0$ & entao custoViews $\leftarrow \mathrm{z} /$ numeroViews \\
senao custoLikes $\leftarrow 1$ & senao custoDislikes $\leftarrow 1$ & senao custoViews $\leftarrow$ numeroViews / $\mathrm{z}$
\end{tabular}


Além das informações de Likes, Dislikes e Views, o custo do Objeto de Aprendizagem também considera a quantidade de conceitos que aquele objeto atende (qtdeConceitos) e, ainda, a quantidade de conceitos que o objeto deixou de atender (custoConteudo). Essas informações são obtidas com base na descrição do vídeo. No processo de recomendação de vídeos da plataforma do YouTube, um objeto potencialmente recomendável será aquele que apresentar custo mínimo. A Equação 7 demonstra que o custo final desse objeto será conduzido pelos atributos dos vídeos, juntamente com o índice de conceitos atendidos.

$$
\text { custoVideo }=\frac{\text { custoLikes }+ \text { custoDislikes }+ \text { custoViews }}{\text { qtdeConceitos }}+\text { custoConteudo }
$$

\section{Resultados}

Para resolver o problema de recomendação de conteúdo foi desenvolvido um Algoritmo Genético com diferentes métodos de seleção (Torneio - TO, Roleta - RO e Ranking - RA), cruzamento (1 ponto - 1P, 2 pontos - 2P e uniforme - UN) e técnicas de elitismo (Melhores - ME, Roleta - RO e Bi-classista - CL). As instâncias de teste foram definidas com 100 OAs e 50 conceitos. Foram realizadas 30 replicações para todas as combinações de operadores genéticos para avaliar a melhor combinação de métodos. Conforme resultados apresentados na Figura 2, as melhores soluções foram obtidas com a utilização do método de seleção de Ranking, cruzamento Uniforme e elitismo com os melhores indivíduo, sendo esta configuração utilizada no decorrer do trabalho.

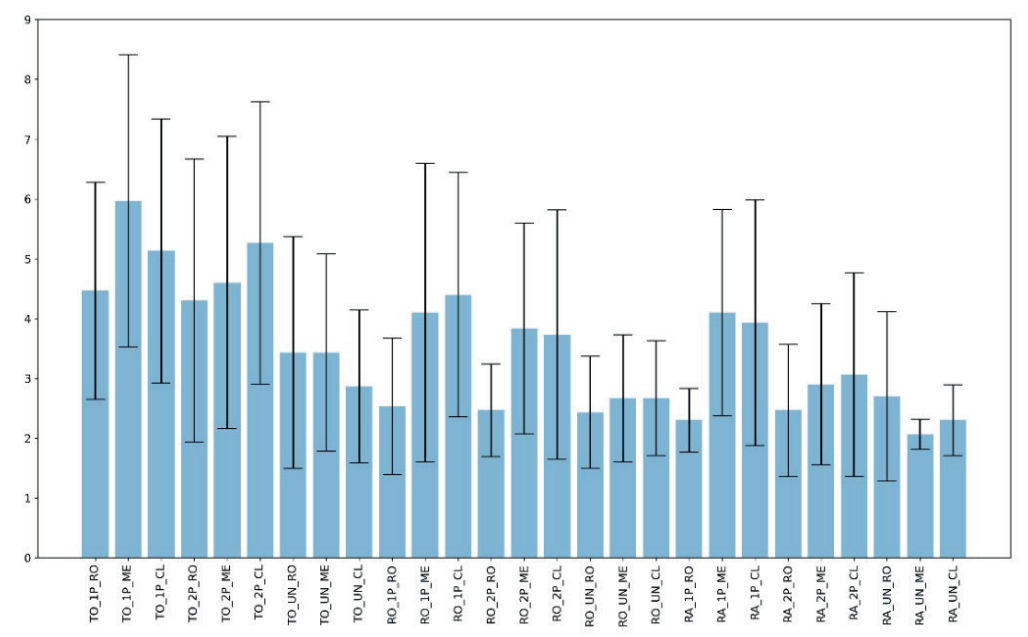

Figura 2. Resultado dos Algoritmos

Para avaliar o modelo min-max do PCC e responder aos questionamentos sobre a qualidade dos objetos de aprendizagem retornados pelo modelo, o algoritmo genético foi executado variando o cálculo da função de aptidão. A primeira (AG_PCC), segue o modelo tradicional do PCC, cuja função objetivo (equação 1) consiste na minimização do custo. A segunda (AG_PCCR), segue o modelo min-max PCC, cuja função objetivo (equação 4) consiste na minimização do custo e do número máximo de repetições de conceitos. Em ambas as versões, o indivíduo que não atende ao conjunto de conceitos requerido, ou seja, indivíduos que representa uma solução inviável, a aptidão foi definida com 0.0001. Assim, espera-se que estes indivíduos sejam, a cada geração, eliminados da população. Neste trabalho consideramos a função de aptidão inversamente proporcional à função objetivo do problema. 
Ambos algoritmos foram executados 30 vezes, considerando três (03) diferentes cenários que variam de acordo com o número de objetos e conceitos. A Tabela 4 apresenta resultados sumarizados. As duas primeiras colunas apresentam as informações da instância avaliada, indicando o número de objetos e o número de conceitos. A $3^{a}$ e $4^{a}$ coluna apresenta a média e desvio padrão (entre parênteses) do critério custo para ambas versões do algoritmo genético. A $5^{a}$ coluna apresenta o percentual de diferença entre os algoritmos considerando o custo. As colunas 6 a 8 apresentam as mesmas informações considerando o critério número máximo de repetições. A última coluna apresenta o custo da solução ótima.

Tabela 4. Comparação entre os algoritmos AG_PCC e AG_PCCR

\begin{tabular}{|c|c|c|c|c|c|c|c|c|}
\hline \multicolumn{2}{|c|}{ Instância } & \multicolumn{2}{|c|}{ Custo } & \multirow{2}{*}{ \%Diferença } & \multicolumn{2}{|c|}{ Num Max Repetições } & \multirow{2}{*}{$\%$ Diferença } & \multirow{2}{*}{$\begin{array}{c}\text { Custo } \\
\text { Sol. Ótima }\end{array}$} \\
\hline$\|$ objetos $\|$ & $\|$ conceitos $\|$ & AG_PCC & AG_PCCR & & AG_PCC & AG_PCCR & & \\
\hline 100 & 20 & $7,1(0,43)$ & $8,6(1,35)$ & $17 \%$ & $4,5(0,63)$ & $3,7(0,65)$ & $18 \%$ & 7 \\
\hline 250 & 50 & $16,0(2,44)$ & $20,0(2$ & $20 \%$ & $7,7(1,09)$ & $5,4(1,04)$ & $30 \%$ & 14 \\
\hline 500 & 100 & $24,3(2,05)$ & $25,8(2,84)$ & $6 \%$ & $8,1(1,04)$ & $7,2(0,74)$ & $11 \%$ & 21 \\
\hline
\end{tabular}

Primeiramente, analisando o critério: minimizar o número máximo de repetições de conceitos, os dados demonstram uma melhora significativa com uso da modelagem proposta neste trabalho, obtendo uma diferença entre $11 \%$ e $30 \%$ de redução no número máximo de conceitos repetidos. Em relação ao critério: minimizar custos dos objetos de aprendizagem, já era esperada uma média melhor do algoritmo AG_PCC, porém observa-se que o AG_PCCR não aumenta significativamente os custos dos objetos de aprendizagem. Nos cenários avaliados, existe uma diferença de até $20 \%$ de aumento nos custos.

Por fim, para demonstrar o uso do sistema de recomendação de objetos de aprendizagem proposto e responder aos questionamentos que se referem a sua aplicação no repositório do YouTube, foi simulada uma pesquisa com o tema "Algoritmos e Estrutura de Dados". As tags que filtram a busca foram: ponteiro, vetor, matriz, lista, pilha, fila, árvore, grafo, repetição, recursividade, aula e exercício.

Dentre os 50 vídeos retornados pela API do YouTube, o sistema recomendou quatro (04) vídeos que atendem aos requisitos do usuário. Destes, a média de likes foi de 1004,75, enquanto dislikes apresentou média de apenas 16,75. Quanto às visualizações, a média de views foi de 36008,5 . Cada objeto retornado aborda em média 2,5 conceitos. O objeto com o maior número de conceitos retornados foi seis (06), e o menor um (01). As repetições dos conceitos dentre os vídeos apresentados ao usuário foi, no máximo, duas (02) repetições.

Evidentemente, o YouTube, tanto em seu uso básico quanto por meio de canais como o YouTube Edu, é um grande fornecedor de conteúdos para aprendizagem. Apesar disso, quando necessária a busca com inúmeros conceitos, o sistema não dispõe de uma organização compreensível desses conceitos no resultado. A lista de vídeos exibida pelo sistema do YouTube não deixa claro onde estão os conceitos que estão sendo buscados pelo usuário, sugerindo vários vídeos com o mesmo conteúdo, obrigando o próprio usuário a fazer esse refinamento do processo de seleção. Os resultados apresentados confirmam que é possível cobrir um número maior de conceitos através de um conjunto menor de objetos (vídeos), recomendados especificamente sobre os conceitos que o aluno deseja consumir. Dessa forma, esta seção apresentou resultados que atestam na eficácia da abordagem apresentada, possibilitando seu uso em ambientes virtuais de aprendizagem como suporte a personalização do processo de ensino-aprendizagem de forma eficiente. 


\section{Conclusões}

Este trabalho apresentou um Sistema para Recomendação de Objetos de Aprendizagem utilizando como base o repositório de vídeos do YouTube. O sistema consiste em receber os conceitos requeridos pelo usuário, fazer uma busca no repositório, selecionar um conjunto mínimo de objetos. Uma das vantagens do sistema proposto é por ser expansivo a diferentes repositórios e não apenas o YouTube. A seleção dos objetos é baseada no Problema de Cobertura de Conjunto, porém este trabalho apresenta uma modelagem min-max, incluindo como critério de otimização a minimização do número máximo de conceitos repetidos. Essa inclusão se mostrou vantajosa por reduzir a carga cognitiva do aluno e evitar desmotivação por rever conceitos já assimilados.

Os resultados mostraram uma redução na repetição dos conceitos nas soluções obtidas. Além disso, o trabalho apresenta diferentes configurações do Algoritmo Genético para solucionar o Problema de Cobertura de Conjunto demonstrando que o método de seleção por Ranking, cruzamento uniforme e elitismo com melhores indivíduos apresentam melhores resultados. Porém, vale ressaltar as dificuldades inerentes do preenchimento da Matriz A (Tabela 1), que relaciona os objetos e conceitos. A plataforma do YouTube não fornece todas as informações em relação aos conceitos abordados nos vídeos, sendo que neste trabalho essa informação foi obtida através das descrições. A aplicação do sistema proposto em outros repositórios, com metadados mais elaborados, pode apresentar resultados mais preciso.

Como sugestão de trabalho futuro pretende-se ampliar o processo de busca de Objetos de Aprendizagem utilizando outros repositórios. Além disso, a função de custo, proposta neste trabalho, é passível de ajustes para uma recomendação de objetos mais criteriosa.

\section{Referências}

AGUIAR, J. J. B.; FECHINE, J. M.; COSTA, E. B. Recomendação de objetos de aprendizagem baseada na popularidade dos objetos e nos estilos de aprendizagem dos alunos. Anais SBIE. CBIE-LACLO, p. 10, 2015.

BARRÉRE, E.; SOUZA, J. F. de; VITOR, M. A.; ALMEIDA, M. A. de. Utilização de enriquecimento semântico para a recomendação automática de videoaulas no moodle. Revista Brasileira de Informática na Educação, v. 28, p. 319, 2020.

BEASLEY, J. E.; CHU, P. A genetic algorithm for the set covering problem. European Journal of Operational Research, p. 94(2):392 - 404, 1996.

BRAGA, J. C.; DOTTA, S.; PIMENTEL, E.; STRANSKY, B. Desafios para o desenvolvimento de objetos de aprendizagem reutilizáveis e de qualidade. DESAFIE! Anais... Curitiba/PR:CEIE/SBC, 2012.

CAZELLA, S. C.; BHEAR, P.; SCHNEIDER, D.; SILVA, K. K. da; FREITAS, R. Desenvolvendo um sistema de recomendação de objetos de aaprendizagem baseado em competências para a educação: relato de experiências. SBIE. CBIE-LACLO, p. 10, 2012.

DAVIDSON, J.; LIEBALD, B.; LIU, J.; NANDY, P.; Van Vleet, T. The YouTube video recommendation system. RecSys'10 - Proceedings of the 4th ACM Conference on Recommender Systems, p. 293-296, 2010. 
FOLDOC. Free On-line Dictionary of Computing: Application program interface. 2015. Disponível em: 〈http://foldoc.org/Application+Program+Interface〉. Acesso em: $23 \mathrm{de}$ setembro de 2020.

GUERRA, P. A. C.; SILVEIRA, S. R.; BERTOLINI, C.; PARREIRA, F. J.; ULBRICHT, V. R. Aplicativo mobile para avaliar a acessibilidade de objetos de aprendizagem utilizando um sistema especialista. Revista Educação Especial, v. 33, p. 32-1, 2020.

JUNIOR, B.; FRANCISCO, C. Reúso de conteúdo da web na recomendação personalizada de objetos de aprendizagem: uma abordagem baseada em um algoritmo genético, tecnologias da web semântica e uma ontologia. Anais SBIE, Uberlândia, Brasil, p. 93, 2018.

JUNQUEIRA, R. D. P.; LOSCIO, B. F. Repositórios de objetos de aprendizagem: uma análise comparativa com ênfase no reúso de conteúdos. Simpósio Brasileiro de Informática na Educação, 2014.

MADDEN, A.; RUTHVEN, I.; MCMENEMY, D. A classification scheme for content analyses of YouTube video comments. Journal of Documentation, v. 69, n. 5, p. 693-714, 2013.

MAVROMMATIS, G. Learning objects and objectives towards automatic learning construction. Eur. J. Oper, p. 10, 2006.

MENDES, M. M.; CARVALHO, V. C.; ARAUJO, R. D.; DORCA, F. A.; CATTELAN, R. G. Clustering Learning Objects in the IEEE-LOM standard considering learning styles to support customized recommendation systems in educational environments. 12th Latin American Conference on Learning Objects and Technologies, LACLO 2017, p. 1-8, 2017.

MOREIRA, M. B.; CONFORTO, D. Objetos de aprendizagem: Discutindo a acessibilidade e a usabilidade. Anais do XXII SBIE - XVII WIE, p. 4, 2011.

NAGUMO, E.; TELES, L. F.; SILVA, L. A. A utilização de vídeos do youtube como

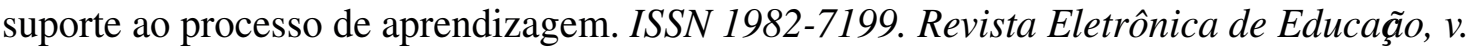
14, 1-12, e3757008, p. 12, 2019.

PINHEIRO, R. R. A. Sistema de recomendação de vídeos educacionais: um estudo de caso no youtube. Dissertaço: Mestrado em Modelagem Computacional de Conhecimento. Universidade Federal de Alagoas, p. 62, 2019.

PONTES, W. L.; FRANA̧, R. M.; COSTA, A. P. M.; BEHAR, P. Filtragens de Recomendação de Objetos de Aprendizagem: uma revisão sistemática do CBIE. Anais do XXV Simpósio Brasileiro de Informática na Educação (SBIE 2014), v. 1, 2014.

SERBANOIU, A.; REBEDEA, T. Relevance-based ranking of video comments on youtube. Proceedings - 19th International Conference on Control Systems and Computer Science, CSCS 2013, IEEE, p. 225-231, 2013.

VIEIRA, F. J. R.; NUNES, A. S. N. Dica: Sistema de recomendação de objetos de aprendizagem baseado em conteúdo. SCIENTIA PLENA, p. 10, 2012.

WILEY, D. A. The Instructional Use of Learning Objects. Bloomington, Indiana: Agency for Instructional Technology, 2002. v. 1.293 p.

YOUTUBE. API Reference. 2019. Disponível em: 〈https://developers.google.com/ youtube/v3/docs/?hl=pt-br $\rangle$. Acesso em: 06 de outubro de 2020.

YOUTUBE. Youtube Insights. 2019. Disponível em: 〈https://youtubeinsights.withgoogle. com $\rangle$. Acesso em: 06 de outubro de 2020. 